\title{
Washing plastic spacers in household detergent reduces electrostatic charge and greatly improves delivery
}

\author{
F. Piérart, J.H. Wildhaber, I. Vrancken, S.G. Devadason, P.N. Le Souëf
}

Washing plastic spacers in household detergent reduces electrostatic charge and greatly improves delivery. F. Piérart, J.H. Wildhaber, I. Vrancken, S. G. Devadason, P.N. Le Souëf. (C) ERS Journals Ltd 1999.

ABSTRACT: Ionic detergents reduce electrostatic charge on plastic spacers, thereby improving in vitro drug delivery. The aim of this study was to gain practical information on the use of detergents and to evaluate the relevance of this information on in vivo drug deposition.

Measurement of electrostatic charge and salbutamol particle size distribution was carried out on detergent-coated and noncoated plastic spacers. The efficiency of four household detergents was compared, and the influence of dilution and the duration of the antistatic effect were studied. In addition, the level of radiolabelled salbutamol deposition in the lungs of eight healthy adults was compared after inhalation through a new versus a detergent-coated spacer.

In vitro, all tested detergents reduced the electrostatic charge on the spacer surface. This resulted in a mean increase of 37.4\% (range 33.5-41.2) in small particle $(<6.8$ $\mu \mathrm{m})$ salbutamol output compared with water-rinsed/drip-dried spacers. Dilution had no influence on the results and the effect lasted for at least four weeks. In vivo, the mean lung deposition of radiolabelled salbutamol in healthy subjects was $45.6 \%$ (range 43.4-49.5) through a detergent-coated spacer compared to $11.5 \%$ (range 7.6 17.9) through a static spacer $(p<0.001)$.

In conclusion, household detergents offer a simple and practical solution to the problem of static on plastic spacers and significantly improve both in vitro and in vivo delivery of salbutamol.

Eur Respir J 1999; 13: 673-678.

The use of pressurized metered-dose inhalers (pMDIs) is associated with a number of problems, including high oropharyngeal deposition and co-ordination difficulties [1]. Holding chambers (spacers) have been designed to overcome these problems [2-5], and are widely used in aerosol therapy for both adults and children.

Due to the inherent nonconducting surface of plastic spacers, electrostatic charge inevitably accumulates on these devices and affects drug output [6-10]. In recent studies, it has been demonstrated that coating spacers with ionic detergent minimizes the static, and thereby improves in vitro drug delivery [8-10]. However, several questions remain. Are commercially-available detergents efficient and at what dilution? What is the duration of the antistatic effect? Is it affected by frequent handling of the spacer?

In addition, very little is known about the influence of electrostatic charge on in vivo drug deposition. There are few deposition studies involving the use of a plastic spacer, and the results are highly variable [11-14]. As the question of electrostatic charge affecting drug delivery from plastic spacers has not been adequately addressed prior to the work of O'CALLAGHAN and coworkers [6,7], there is a strong possibility that a substantial part of the variability between studies using similar spacer devices and breathing patterns is due to differences in electrostatic charge. The effect of reducing electrostatic charge on the lung deposition from a spacer has not been adequately quantified.
Perth Medical Aerosol Research Group, Dept of Respiratory Medicine, Princess Margaret Hospital for Children, Perth, Western Australia.

Correspondence: F. Piérart

Service de Pédiatrie

Clinique Notre-Dame de Grâce

212, Chaussée de Nivelles

6041 Gosselies

Belgium

Fax: 3271379481

Keywords: Aerosol therapy

deposition

electrostatic charge

plastic spacers

Received: December 41997

Accepted after revision August 151998

This study was partially funded by GlaxoWellcome, UK.

\section{Materials and methods}

\section{Study design}

In vitro studies. Measurements of electrostatic charge and delivery of salbutamol (Ventolin $\AA$; Allen \& Hanbury's, Sydney, NSW, Australia) were carried out on Volumatic $($ S spacers (Allen \& Hanbury's), which were divided into two groups.

In the first group, non-detergent-coated spacers which were either: 1) new spacers, which had been stored in their original plastic bag; or 2) water-rinsed/drip-dried spacers (treatment recommended by the manufacturer).

In the second group, detergent-coated spacers were treated as shown in table 1. Different detergents were compared regarding their "antistatic property", and the following variables were studied: influence of dilution, duration of effect, and influence of handling. 
Table 1. - Treatment of detergent coated spacers for in vitro study

\begin{tabular}{lcccc}
\hline $\begin{array}{l}\text { Variable to } \\
\text { be tested }\end{array}$ & Detergent & Dilution & $\begin{array}{c}\text { Time } \\
\text { between } \\
\text { coating and } \\
\text { testing }\end{array}$ & Handling \\
\hline Choice of & A & $1: 125$ & $12-24 \mathrm{~h}$ & $\begin{array}{r}\text { Without } \\
\text { handling }\end{array}$ \\
$\begin{array}{l}\text { detergent/ } \\
\text { influence of } \\
\text { dilution }\end{array}$ & B & $\begin{array}{l}1: 1250 \\
1: 2500\end{array}$ & & \\
& D & $1: 5000$ & & \\
$1: 7500$ & & \\
$\begin{array}{l}\text { Duration of } \\
\text { effect/ }\end{array}$ & D & $1: 10000$ & Week 0 (24 h) & Without \\
influence & & & $\begin{array}{l}\text { Week 1 } \\
\text { Week 2 }\end{array}$ & and with \\
of handling & & & Week 3 & \\
& & & Week 4 & \\
\hline
\end{tabular}

Detergents used were: A: Cetrimide; B: Farmland; C: Sunlight; D: Palmolive.

All experiments were carried out in controlled laboratory conditions. The mean temperature was $23^{\circ} \mathrm{C}$ (range $22-24$ ) and the mean relative humidity was $62 \%$ (range 56-70).

In vivo study. Eight healthy nonsmoking volunteers (five males) aged between 25 and 42 yrs were recruited. All participants gave written informed consent. They had normal predicted values for forced vital capacity (FVC) (mean $103.2 \%$, range $85-112$ ) and forced expiratory volume in one second (FEV1) (mean 100.5\%, range 87111). The study was approved by the local hospital Ethics Committee.

All subjects attended the department on two occasions, at least $48 \mathrm{~h}$ apart. On each visit, in random order, they were asked to inhale a $500 \mu \mathrm{g}$ dose $(5 \times 100 \mu \mathrm{g})$ of technetium-99m-labelled salbutamol through either a high electrostatic (new) or a low electrostatic (detergent-coated) Volumatic spacer. Prior to testing, they were instructed on the use of the pMDI and the spacer, and were trained using a Vitalograph Compact Spirometer (Vitalograph Ltd, Buckingham, UK) to produce a slow inhalation with a flow of 20-25 L.min ${ }^{-1}$. The inhaler was first shaken for $30 \mathrm{~s}$ and primed before use. The subjects were asked to exhale and, after actuation of the pMDI through the spacer, they performed a slow maximal inhalation. They held their breath for $10 \mathrm{~s}$ and then expired through a filter (Curityß) Anesthesia Filter; Kendall, MA, USA). The inhaler was shaken between each actuation. For each subject, it took $90-120 \mathrm{~s}$ to inhale the five actuations. Following administration of the radiolabelled drug, subjects were asked to rinse their mouths and gargle with water. The liquids from the mouthwash and gargle were collected separately. Immediately after inhalation, imaging procedures were carried out using a gamma camera.

\section{Methods}

Detergent coating. The spacers were soaked for a few minutes in the appropriate dilution of a detergent solution and allowed to drip-dry for $12-24 \mathrm{~h}$. Four ionic detergents were tested in vitro (A: Cetrimide $\mathrm{R}$ ), cationic, Ramprie Laboratories, Perth, WA, Australia; B: Farmland $\mathbb{R}$, anionic, Coles Supermarkets, Melbourne, Victor- ia, Australia; C: Sunlight $\mathbb{R}$, anionic, Lever Rexona, Sydney, NSW, Australia; D: Palmolive R, anionic, Colgate-Palmolive, Sydney, NSW, Australia). The spacers used for the in vivo study were coated with Palmolive (1:5,000 dilution).

Handling. Handling was performed by manually actuating salbutamol (2 puffs of $100 \mu \mathrm{g}$ ) twice a day in a group of coated spacers for the stated period of time. After each actuation, a continuous flow of $60 \mathrm{~L} \cdot \mathrm{min}^{-1}$ was applied for $5 \mathrm{~s}$.

Measurement of electrostatic charge on spacers. Quantitative measurement of electrostatic charge on each spacer was performed using an electrometer (Model 37c; Electronic Instruments Ltd, Jacoby Mitchell, Sydney, NSW, Australia). Charge was measured along the inner surface of each spacer piece.

Surface charge density was classified into three categories: negligible $\left(0-1.2 \mu \mathrm{C} \cdot \mathrm{m}^{-2}\right)$, low $\left(1.2-3.3 \mu \mathrm{C} \cdot \mathrm{m}^{-2}\right)$ or high $\left(3.3-6.7 \mu \mathrm{C} \cdot \mathrm{m}^{-2}\right)$.

Assessment of particle size distribution. Particle size distribution from salbutamol inhalers through the spacers was measured using a Multistage liquid impinger (MSLI; Copley, Nottingham, UK) with a continuous flow of $60 \mathrm{~L} \cdot \mathrm{min}^{-1}$. The pMDI was shaken for $30 \mathrm{~s}$ and two actuations were made prior to testing. Ten single actuations were introduced into the spacer, which was attached to the MSLI. The pMDI was shaken between actuations. Drug particles were deposited on the actuator, the spacer, the glass throat, or one of four stages according to their size. The particle size classifications for stages $1,2,3$ and 4 were $>13 \mu \mathrm{m}, 6.8-13 \mu \mathrm{m}, 3.1-$ $6.8 \mu \mathrm{m}$ and $<3.1 \mu \mathrm{m}$, respectively. The actuator, spacer, glass throat and stages of the MSLI were washed with $45 \mathrm{~mL}$ methanol, and $5 \mathrm{~mL} 0.1 \mathrm{M} \mathrm{NaOH}$ was added to each wash. The absorbance (246 nm) of each sample was measured in duplicate using an ultraviolet spectrophotometer (Hitachi U-2000; Tokyo, Japan). The concentration of salbutamol was calculated using a standard curve obtained with known concentrations of salbutamol measured in $45 \mathrm{~mL}$ methanol and $5 \mathrm{~mL} 0.1 \mathrm{M} \mathrm{NaOH}$. The standard curve was linear $\left(\mathrm{r}^{2}=1.00\right)$ for concentrations between 0 and $20 \mu \mathrm{g} \cdot \mathrm{mL}^{-1}$. Each experiment was repeated four times for each different type of spacer.

Preparation of radiolabelled metered dose inhalers. Salbutamol (Ventolin(R) was labelled with ${ }^{99 \mathrm{~m}} \mathrm{Tc}$ following the method of KöHLER et al. [15], with some modifications. Technetium pertechnetate $\left({ }^{99 \mathrm{~m}} \mathrm{TcO}_{4}{ }^{-} ; 500-600\right.$ $\mathrm{MBq}$ ) was eluted from a radionuclide generator (Australian Radioisotopes, Lucas Heights, NSW, Australia) and added to $4 \mathrm{~mL}$ butanone in a separating funnel. After shaking vigorously, the two phases were allowed to separate. The top layer (organic phase) was collected, poured into an empty canister, and carefully evaporated to dryness using a low flow of nitrogen $\left(9-13 \mathrm{~L} \cdot \mathrm{min}^{-1}\right)$. After all solvent had evaporated, the canister was placed on a hot plate for $30 \mathrm{~min}$, at a temperature between 73 and $85^{\circ} \mathrm{C}$, and subsequently cooled in dry ice. The contents of a cooled commercial salbutamol pMDI were then poured into the radiolabel-containing canister, a metering valve was added, and the canister was immediately sealed by a crimper (type $555 \mathrm{G}$, Pamasol; Willi 
Mäder, Pfäffikon Switzerland). The canister was placed on a shaker for $30 \mathrm{~min}$, and was then ready for testing.

The exact dose of activity per actuation was determined by placing the labelled canister in an ionization chamber (Atomlab 200 dose calibrator; Gammasonics, Sydney, NSW, Australia) both before and after actuation of five puffs.

Validation of radiolabelling technique. Accuracy of labelling was confirmed using a MSLI to ensure that the label followed the drug and that the radiolabelling method did not significantly alter the particle size distribution of the aerosol generated by the salbutamol pMDI. The particle size distribution of commercial salbutamol pMDIs was compared to that of both drug and radiolabel from the labelled inhalers. For the labelled pMDIs, the distribution of radioactivity in the different washes (actuator, glass throat, stages 1-4) was measured in an ionization chamber (Atomlab 200 dose calibrator). On the study days, the particle size distribution from the labelled inhaler was assessed prior to administration to confirm that the labelling was satisfactory. These measurements were repeated after the studies to make sure that no change had occurred.

Lung function tests. As normal lung function was an inclusion criterion, FVC and FEV1 were measured in all participants, using a PneumoCheck Spirometer (61000; Welch Allyn, Skaneateles Falls, NY, USA).

Imaging procedures. A flood source containing approximately $37 \mathrm{MBq}{ }^{99 m} \mathrm{Tc}$ was used to obtain individual values for the attenuation of activity due to absorption by body tissues, as previously described. [16, 17]. Immediately after inhaling the ${ }^{99 \mathrm{~m}}$ Tc-labelled salbutamol, anterior and posterior images of the chest and abdomen were obtained together with lateral views of the upper airways using a gamma camera (Dual Head GCA-7200 A; Toshiba, Tochigi-ken, Japan). Collection times were 2 min for each image. Separate count rates were determined for the right and left lungs, mouth, throat, oesophagus and stomach. Each count rate was corrected for background counts and attenuation, and the geometric means of corresponding anterior and posterior count rates were calculated. The spacer, the actuator, the mouth and gargle washings, and the expiratory filter were also imaged. All deposition percentages were calculated from the total radioactivity given.

Analysis. Statistical analysis was carried out using version 5a of the Microsoft Excel Analysis ToolPak (Microsoft Corporation, Redwood, WA, USA), using analysis of variance (ANOVA) for repeated measurements. A p-value $<0.05$ was considered significant.

\section{Results}

In vitro studies

Electrostatic charge was negligible $\left(<1.2 \mu \mathrm{C} \cdot \mathrm{m}^{-2}\right)$ on the surface of all detergent-coated spacers, regardless of the brand of detergent or the dilution used, and high $(>5$ $\mu \mathrm{C} \cdot \mathrm{m}^{-2}$ ) on all noncoated spacers.

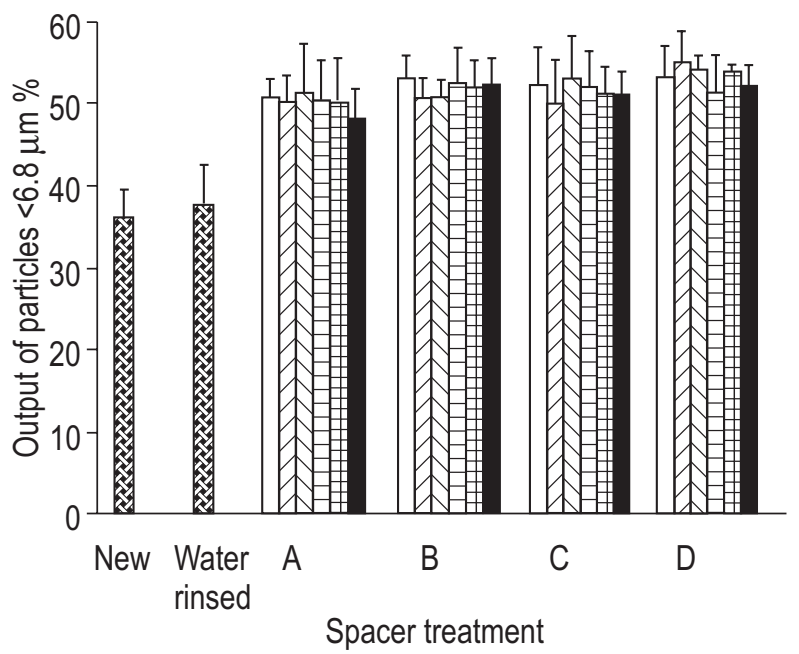

Fig. 1. - Small particle $(<6.8 \mu \mathrm{m})$ output of salbutamol pressurized metered-dose inhalers through new and water-rinsed/drip-dried spacers compared to that through spacers coated with different detergents (A: Cetrimide $(\mathrm{A}$; B: Farmland $(\mathrm{R} ;$ C: Sunlight $(\mathrm{R} ; \mathrm{D}$ : Palmolive $(\mathrm{R})$ using six different dilutions $(\square: 1 / 125 ; \mathbb{Z}: 1 / 1,250 ; \mathbb{\mathbb { Q }}: 1 / 2,500$; 冒 $: 1 / 5,000 ;$ 曲: 1 / $7,500$; and $\square: 1 / 10,000)$. Values are presented as mean \pm SD.

Using coated spacers, the mean (SD) small particle $(<6.8$ $\mu \mathrm{m})$ salbutamol output ranged from $50.1 \%$ (4.0) using detergent A to 53.1\% (3.1) using detergent D. There was no significant difference between the four detergents tested. This mean output was significantly greater in comparison with that from the new spacers $(36.2 \%(3.5) ; \mathrm{p}<$ $0.001)$, and the water-rinsed/drip-dried spacers $(37.6 \%$ (5.1); p<0.002) (fig. 1).

All dilutions gave similar results, regardless of the detergent used. Even using a spacer coated with very dilute detergent $(1: 10,000)$, the small particle output of salbutamol was significantly higher than that from a water-rinsed/ drip-dried spacer $(p<0.05$ for detergent $A ; p<0.005$ for detergents B, C and D) (fig. 1).

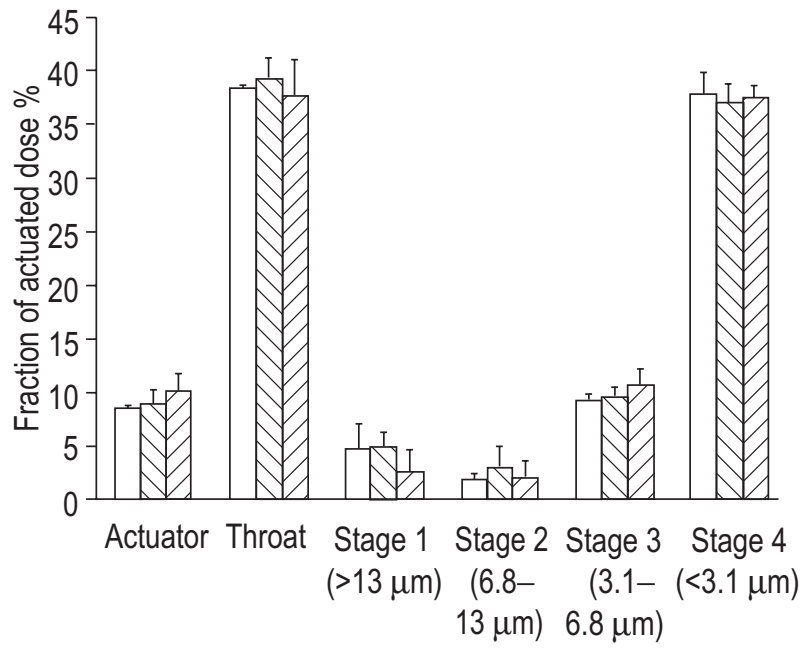

Fig. 2. - Particle size distribution of drug from commercial and labelled salbutamol pressurized metered-dose inhalers (pMDIs) and radiolabel distribution from labelled salbutamol pMDIs. (Multistage liquid impinger with a continuous flow of $60 \mathrm{~L} \cdot \mathrm{min}^{-1}$ ). Values are presented as mean and SD. $\square$ : Unlabelled drug; $\mathbb{\mathbb { V }}$ : labelled drug; $\mathbb{Z}$ : radiolabel. 


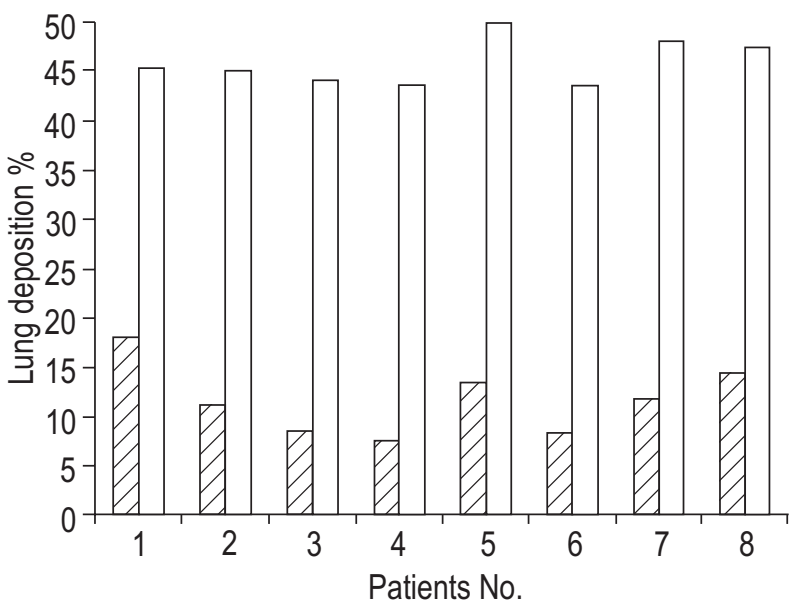

Fig. 3. - Comparison of salbutamol lung deposition, expressed as percentage of total actuated dose, after inhalation of salbutamol through electrostatically charged (new; $\mathbb{Z}$ ) and non-electrostatically charged (detergent-coated; $\square$ ) Volumatic spacers in eight healthy adults.

With or without handling, electrostatic charge became detectable 1 week after coating but remained in the low range $\left(1.2-3.3 \mu \mathrm{C} \cdot \mathrm{m}^{-2}\right)$ for at least 4 weeks. However, the small particle output of salbutamol did not significantly change: without handling, the mean (SD) output was $51.2 \%$ (0.6) at week 0 and $47.9 \%$ (4.7) at week 4; with handling, the mean (SD) output was $47.9 \%$ (5.3) at week 0 and $46.6 \%$ (6.3) 4 weeks after coating.

\section{In vivo study}

The particle size distribution of salbutamol pMDIs was compared to the distribution of both drug and radiolabel from the labelled inhalers as part of the method validation (fig. 2). There was a good correlation between label and drug delivery, particularly for stages 3 and 4: the mean output of particles $<6.8 \mathrm{~mm}$, expressed as a percentage of the metered dose, was $46.7,46.2$ and $47.7 \%$ for unlabelled drug, labelled drug and radiolabel, respectively.

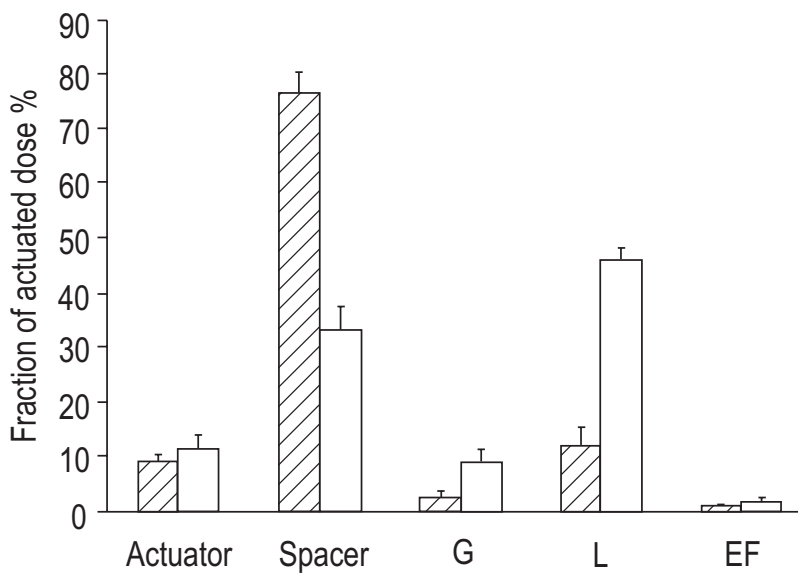

Fig. 4. - Relative deposition of salbutamol, expressed as a percentage of total actuated dose, in the actuator, spacer, gastrointestinal tract (mouth, throat, oesophagus and stomach $(\mathrm{G})$ ), lungs (L) and expiratory filter (EF), after inhalation through electrostatically charged (new; $\mathbb{Z}$ ) and non-electrostatically charged (detergent-coated; $\square$ ) Volumatic spacers in eight healthy adults. Values are presented as mean and SD.

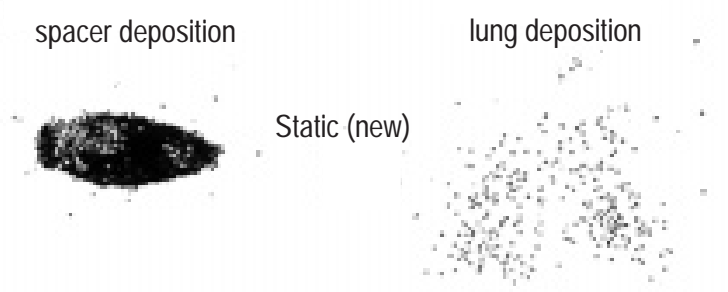

Nonstatic (detergent-coated)

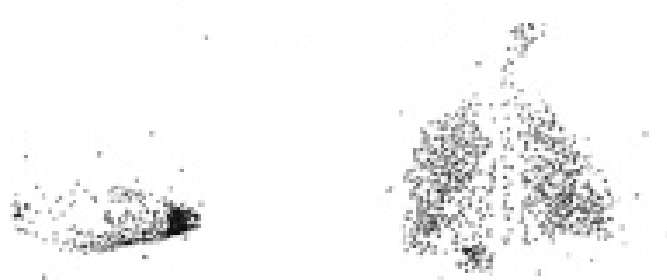

Fig. 5. - Typical deposition patterns of radioaerosol in the same subject after inhalation through a new and a detergent-coated spacer (posteroanterior view).

The radiolabelled doses were reproducible. With a separate canister on each of four study days a 1.9-2.1 MBq (mean $2.0 \mathrm{MBq}$ ) dose and a 520-570 $\mu \mathrm{g}$ salbutamol dose (mean $545 \mu \mathrm{g}$ ) per five actuations were obtained.

Electrostatic charge was high $\left(>5 \mu \mathrm{C} \cdot \mathrm{m}^{-2}\right)$ on the surface of all new spacers and negligible $\left(<1.2 \mu \mathrm{C} \cdot \mathrm{m}^{-2}\right)$ on all detergent-coated spacers. Comparison of lung deposition of salbutamol after inhalation through an electrostatic and non-electrostatically charged spacer is shown for each subject in figure 3.

Figure 4 summarizes the deposition, expressed as a percentage of total actuated dose, in the actuator, spacer, gastrointestinal tract (mouth, throat, oesophagus, stomach, mouth washing, gargle washing), lungs and expiratory filter. There was a three-fold increase in lung deposition from detergent-coated spacers (mean $45.6 \%$ (2.2); range 43.4-49.5) compared to static spacers (mean $11.5 \%$ (3.5); range 7.6-17.9). The mean (range) gastrointestinal deposition was $2.4 \%(1.0-3.9)$ through a static spacer and $8.8 \%(4.1-11.2)$ through a nonstatic spacer $(\mathrm{p}<0.001)$. The mean (range) amount of salbutamol remaining in the static spacers was $76.7 \%$ (71.8-81.4) compared to $33.1 \%(26.0-40.8)$ in the detergent-coated spacers $(\mathrm{p}<0.001)$. Typical deposition patterns are shown in figure 5.

\section{Discussion}

The present study demonstrated that reducing the electrostatic charge on a plastic spacer significantly improved both in vitro and in vivo drug delivery. The magnitude of the in vivo increase was remarkably high.

The in vitro studies confirm and extend the authors' previous work $[8,10]$. Coating a plastic spacer with an ionic detergent is a simple method of reducing electrostatic charge, thereby increasing the in vitro drug delivery. The four detergents tested gave similar results: a mean increase of $37.4 \%$ in the output of small particles $(<6.8 \mu \mathrm{m})$ when using coated spacers compared to water-rinsed/drip-dried spacers. Most commercially-available dishwashing liquids 
are made up of ionic (mainly anionic) detergents. Therefore, the present study would suggest that similar results may be obtained for most household detergents. All dilutions of the detergents were equally effective. The antistatic effect lasted for at least four weeks, even with repeated handling of the spacer.

The in vivo study demonstrates that electrostatic charge plays an important role in the lung deposition of salbutamol through plastic spacers, and that the effect of reducing charge is much greater than expected. Using detergent-coated Volumatic spacers, a mean lung deposition of salbutamol of $45.6 \%$ was found, which was much higher than in previous studies [11-14, 18, 19].

Most deposition studies using radiolabelled salbutamol have examined the use of pMDIs, dry powder inhalers, or nebulizers, and have reported mean lung deposition percentages between 9 and $21.6 \%[11,18,19]$. There are few deposition studies involving the use of a spacer device, and the results of these studies are highly variable. TAL et al. [12] evaluated the deposition of directly radiolabelled salbutamol in two adult volunteers after inhalation through an Aerochamber $\AA$, and found a mean lung deposition of $19 \%$. However, their study refers to deposition in children rather than in adults, and the deposition values in that study are typically lower. NEWMAN et al. [13] reported a mean lung deposition of $16.1 \%$ sodium cromoglycate in 10 healthy volunteers using an open spacer device (Syncroner $\mathbb{R}$ ).

Using the Volumatic, MELCHOR et al. [11] reported a mean deposition of $20.9 \%$ labelled salbutamol in the lungs of 10 normal subjects, and a mean of $19.0 \%$ in the lungs of 19 asthmatic patients. MatTHYs et al. [14] found a mean total lung deposition of $34 \%$ labelled salbutamol in four normal subjects. In both studies, there was no information regarding the treatment of the spacers or the level of electrostatic charge on their surface. A substantial part of the variability between studies using the same spacer device and the same breathing pattern may be due to differences in electrostatic charge. Another factor contributing to this variability could be the quality of the radiolabelling technique.

In the present study, the mean (range) lung deposition after inhalation of salbutamol through a static spacer was particularly low: $11.5 \%$ (7.6-17.9). This finding could be explained by two factors: firstly new spacers were used which had been stored in their original plastic bag and therefore had very high levels of electrostatic charge; secondly, all the subjects inhaled at a low flow rate (20-25 $\left.\mathrm{L} \cdot \mathrm{min}^{-1}\right)$, which could have increased the influence of static by prolonging the contact time between the aerosol cloud and the walls of the spacer. Effects of various inhalation modes on the deposition of radioactive aerosols are well known [20], but previous in vitro studies have demonstrated that the influence of lower flow rates and delay was greatly reduced or even eliminated by reducing the charge on the spacer, and that the use of non-electrostatically charged spacers leads to a higher and more predictable dose delivery $[10,21]$.

Coating a plastic spacer with detergent increased the total drug output through the spacer. Hence, the gastrointestinal deposition was also increased. However, using low electrostatically charged spacers, it may be possible to reduce the nominal dose prescribed to patients, while retaining a similar therapeutic effect. The clinical significance in terms of bronchodilator response was not assessed in this study. This increased delivery would be of greater relevance to the use of inhaled corticosteroids. Further studies could be performed to confirm that the results produced in this study correlate with an improved therapeutic ratio.

The drug delivery from spacer devices depends on different factors, such as spacer volume, electrostatic charge, type of valve, dead space, spacer emptying pattern and mode of inhalation breathing $[22,23]$. However, the authors' previous in vitro work $[8,10]$ has demonstrated that detergent coating improves the delivery of salbutamol

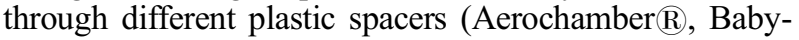

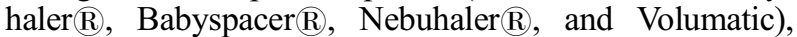
confirming that electrostatic charge plays a major role. A low-volume metal spacer [21] has been developed to eliminate the problem of static charge, but in view of the present results and the issues of cost and availability, a simple plastic spacer may remain the device of choice worldwide.

The authors' studies were performed using chlorofluorocarbon (CFC)-salbutamol, but previous studies have demonstrated that the electrostatic charge affects the delivery of other drugs from plastic spacers, such as sodium cromoglycate [6] and budesonide [7]. Further studies with hydroxyfluroalkane (HFA) formulations are needed.

The information obtained in this study can be used to optimize the delivery of most inhaled medications from plastic spacers.

In conclusion, the coating of plastic spacers with household detergents significantly improves both in vitro and in vivo salbutamol delivery. The present in vitro studies suggest that most commercially-available detergents may do this efficiently, even at low concentrations, and that the antistatic property of detergent coating lasts for at least 4 weeks. The present in vivo study confirms that detergents offer a practical solution to the problem of electrostatic charge on plastic spacers and improve lung deposition of salbutamol much more than expected. The variability of deposition is also reduced. In addition, these studies allow us to provide practical advice on the care of all plastic spacers. It is recommended that plastic spacers should be soaked in a dilute solution of household detergent and then allowed to drip-dry without water-rinsing. This treatment should be repeated at least once a month.

Further studies are needed to extend the scope of these findings to the use of inhaled corticosteroids in asthmatic patients and to assess the clinical relevance of this information. However, the present results indicate that detergent-coating of plastic spacers is a simple way of providing a greater and more predictable delivery of drug to the airways, and thus, may indicate the potential for dose reduction of inhaled medications.

Acknowledgements. We are indebted to $\mathrm{R}$. Troedson, (Nuclear Medicine Department), and C. Roller, J.M. Wilson, T. Lagana and B.C. Thompson for their technical assistance.

\section{References}

1. Everard ML, Devadason SG, Summers QA, Le Souëf PN. Factors affecting total and "respirable" dose delivered by a salbutamol metered dose inhaler. Thorax 1995; 50: 746749. 
2. Selroos O. Bronchial asthma, chronic bronchitis and pulmonary parenchymal diseases. In: Moren F, Dolovich MB, Newhouse MT et al., eds. Aerosols in Medicine: Principles, Diagnosis and Therapy. Amsterdam, Elsevier, 1993; pp. 261-289.

3. Selroos O, Pietmalho A, Riska H. Delivery devices for inhaled asthma medication. Clinical implications of differences in effectiveness. Clin Immunother 1996; 6: 273299.

4. Selroos O, Halme M. Effect of a volumatic spacer and mouth rinsing on systemic absorption of inhaled corticosteroids from a metered dose inhaler and dry powder inhaler. Thorax 1991; 46: 891-894.

5. Barry $\mathrm{PW}, \mathrm{O}^{\prime}$ Callaghan C. Inhalational drug delivery from seven different spacer devices. Thorax 1996; 51: $835-840$.

6. O'Callaghan C, Lynch J, Cant M, Robertson C. Improvement in sodium cromoglycate delivery from a spacer device by use of an antistatic lining, immediate inhalation, and avoiding multiple actuations of drug. Thorax 1993; 48: 603-606.

7. Barry PW, $\mathrm{O}^{\prime}$ Callaghan $\mathrm{C}$. The effect of delay, multiple actuations and spacer static charge on the in vitro delivery of budesonide from the Nebuhaler. Br J Clin Pharmacol 1995; 40: 76-78.

8. Wildhaber JH, Devadason SG, Hayden MJ, et al. Electrostatic charge on a plastic spacer device influences the delivery of salbutamol. Eur Respir J 1996; 9: 1943-1946.

9. Clark DJ, Lipworth BJ. Effect of multiple actuations, delayed inhalation and antistatic treatment on the lung bioavailability of salbutamol via a spacer device. Thorax 1996; 51: 981-984.

10. Wildhaber JH, Devadason SG, Eber E, et al. Effect of electrostatic charge, flow, delay and multiple actuations on the in vitro delivery of salbutamol from different small volume spacers for infants. Thorax 1996; 51: 985-988.

11. Melchor R, Biddiscombe MF, Mak VHF, Short MD, Spiro SG. Lung deposition patterns of directly labelled salbutamol in normal subjects and in patients with reversible airflow obstruction. Thorax 1993; 48: 506-511.

12. Tal A, Golan H, Grauer N, Aviram M, Albin D, Quastel MR. Deposition pattern of radiolabelled salbutamol in- haled from a metered-dose inhaler by means of a spacer with mask in young children with airway obstruction. $J$ Pediatr 1996; 128: 479-484.

13. Newman SP, Clark AR, Talaee N, Clarke SW. Pressurised aerosol deposition in the human lung with and without an "open" spacer device. Thorax 1989; 44: 706-710.

14. Matthys H, Eltschka R, App EM. Deposition pattern of a radioactively labelled $\beta_{2}$ sympathomimetic aerosol. Atemw Lungenkrkn 1988; 14: 485-488.

15. Köhler D, Fleischer W, Matthys H. New method for easy labelling of $\beta_{2}$ agonists in the metered dose inhaler with technetium-99m. Respiration 1988; 53: 65-73.

16. Maccy DJ, Marshall R. Absolute quantification of radiotracer uptake in the lungs using a gamma camera. $J$ Nucl Med 1982; 23: 731-734.

17. Forge NI, Mountford PJ, O'Doherty MJ. Quantification of technetium-99m lung radioactivity from planar images. Eur J Nucl Med 1993; 20: 10-15.

18. Zainudin BMZ, Biddiscombe M, Tolfree SEJ, Short M, Spiro SG. Comparison of bronchodilator responses and deposition patterns of salbutamol inhaled from a pressurised metered dose inhaler, as a dry powder, and as a nebulised solution. Thorax 1990; 45: 469-473.

19. Farr SJ, Rowe AM, Rubsamen R, Taylor G. Aerosol deposition in the human lung following administration from a microprocessor controlled pressurised metered dose inhaler. Thorax 1995; 50: 639-644.

20. Newman SP, Pavia D, Garland N, Clarke SW. Effects of various inhalation modes on the deposition of radioactive pressurised aerosols. Eur J Respir Dis 1982; 63 (Suppl. 119): 57-65.

21. Bisgaard H, Anhoj J, Klug B, Berg E. A non-electrostatic spacer for aerosol delivery. Arch Dis Childhood 1995; 73: 226-230.

22. Barry PW, $\mathrm{O}^{\prime}$ Callaghan $\mathrm{C}$. The output of salbutamol from spacer devices. Am J Respir Crit Care Med 1997; 155: A671.

23. Devadason SG, Wildhaber JH, Linto JM, Summers QA, Le Souëf PN. Factors affecting in vitro delivery from plastic spacers. Am J Respir Crit Care Med 1996; 153: A60. 